\title{
Guillain-Barré Syndrome-Like Polyneuropathy Associated with Immune Checkpoint Inhibitors: A Systematic Review of 33 Cases
}

\author{
Yan Li, Xiuchun Zhang, and Chuansheng Zhao \\ Department of Neurology, The First Hospital of China Medical University, Shenyang 110001, China \\ Correspondence should be addressed to Chuansheng Zhao; cszhao@cmu.edu.cn
}

Received 10 April 2021; Revised 8 June 2021; Accepted 5 August 2021; Published 19 August 2021

Academic Editor: Steven De Vleeschouwer

Copyright ( 2021 Yan Li et al. This is an open access article distributed under the Creative Commons Attribution License, which permits unrestricted use, distribution, and reproduction in any medium, provided the original work is properly cited.

\begin{abstract}
Immune checkpoint inhibitors (ICIs) have been increasingly used in the treatment of various types of tumors with favorable results. But these treatments also led to a variety of immune-related adverse events (irAEs). Neurological irAEs such as Guillain-Barré Syndrome are rare and may have serious consequences once they occur. A systematic literature search was performed in PubMed and Embase for all case reports of GBS associated with ICIs published in English reporting on human beings from 1990 up to date. A total of 30 case reports (total patients =33) were used for final analysis. The included cases were from 11 countries, covering 10 tumor types, with melanoma accounting for the largest number. The mean age was 62.2 \pm 11.1 years old, and males were dominant (male: 26 and female: 7). The median time of initial symptoms was 8.2 weeks after the $1^{\text {st }}$ dose of ICIs. The most common manifestations of GBS associated with ICIs were weakness, hyporeflexia or areflexia, and paresthesia in order. The GBS subtypes suggested by electrophysiological results were acute inflammatory demyelinating polyneuropathy (AIDP), acute motor axonal neuropathy (AMAN), and Miller Fisher syndrome (MFS). The protein level of CSF in patients with GBS related to ICIs was $180.68 \pm 152.51 \mathrm{mg} / \mathrm{dl}$. Immediate termination of ICIs followed by intravenous immunoglobulin was the preferred treatment option. $72.7 \%$ of patients recovered or had residual mild dysfunction after treatment. Elderly male patients with melanoma were most likely to develop ICI-related GBS. The specific neurological symptoms, CSF analysis, and electrophysiological examination were important means of diagnosis.
\end{abstract}

\section{Introduction}

In the last decade, with a better understanding of the factors that promote or inhibit $\mathrm{T}$ cell response, great progress has been made on tumor immunotherapy. Immune checkpoint inhibitors (ICIs) have become a powerful clinical strategy for treating cancer, including an antibody targeting cytotoxic T lymphocyte-associated antigen-4 (CTLA-4, e.g., ipilimumab), antibodies directed against programmed cell death protein-1 (PD-1, e.g., nivolumab, pembrolizumab, and cemiplimab), and anti-PD-1 ligand (PD-L1, e.g., atezolizumab, durvalumab, and avelumab) [1]. These drugs can be used alone or in combination with other immunotherapy [2] or chemotherapy [3] to improve the survival of cancer patients. Currently, it has been used for the treatment of tumors of lung, kidney, liver, bladder and breast cancer, melanoma, and lymphomas [4-6]. ICIs improve the prognosis and qual- ity of life of patients, whereas the increase of use also brings various immune-related adverse events (irAEs). The dermatologic, gastrointestinal, pulmonary, hepatic, and endocrine systems were most frequently involved.

Cases of neurological irAEs are rare, accounting for less than 3\% [7]. So far, the best-characterized central nervous system irAEs are encephalitis and meningitis. And neurologic irAEs known to be most relevant to the peripheral nervous system are peripheral neuropathies, GBS, myasthenia gravis, and myositis [8]. Once they occur, such as encephalitis, Guillain-Barré syndrome, or myasthenia gravis, they can develop into serious consequences or even death.

Epidemiology shows that nearly two-thirds of patients with GBS have a recent history of infection before the illness [9]. GBS with potentially life-threatening consequences occurs in approximately $0.1-0.2 \%$ of patients treated with ICIs [10]. To date, information on the incidence, 
characteristics, and outcomes of GBS associated to ICIs treatment is very limited. And the available information varies widely in diagnosis and treatment. Multidisciplinary treatment of tumors urgently requires neurologists and oncologists to accurately understand the clinical manifestations and treatment of ICI-related GBS.

This review summarized the published data on GBS or GBS-like disease occurring in patients after treatment with ICIs from 1990 up to date and analyzed their time patterns of occurrence, clinical presentation, diagnosis, treatment, and prognosis.

\section{Methods}

A systematic literature search was performed in PubMed and Embase for all case reports of GBS associated with ICIs published in English reporting on human beings from 1990 up to date. For the case reports search, the keywords used were as follows: ["Guillain-Barré Syndrome" OR "acute inflammatory demyelinating polyradiculoneuropathy" OR "Miller Fisher Syndrome" OR "acute motor axonal neuropathy" OR "acute motor-sensory axonal neuropathy"] AND ["Immune Checkpoint Inhibitors" OR "Immune Checkpoint Blockers" OR "PD-L1 Inhibitors" OR "Programmed DeathLigand 1 Inhibitors" OR "CTLA-4 Inhibitors" OR "Cytotoxic T-Lymphocyte-Associated Protein 4 Inhibitors" OR "PD-1 Inhibitors" OR "Programmed Cell Death Protein 1 Inhibitor"]. Abstracts of medical conference were excluded. For each case, we extracted data on demographics and clinical manifestations and adjuvant examinations (imaging, cerebrospinal fluid, and electrophysiology). If gender, age, GBS clinical variation [11], electrophysiological subtype [12], or results of the relevant examination were not explicitly reported in the article, this case could not be considered for analysis. The search was conducted by Yan Li and Xiuchun Zhang. The selection of the articles should be agreed upon by the above two persons.

GraphPad Prism 8 was used for statistical analysis, and continuous data were expressed in the form of mean \pm standard deviation or median. $P<0.05$ was considered statistically significant. Since the proportion of males and females in the included cases was significantly different, unpaired $t$ -test was used to analyze the ages of males and females.

Because the patient's personal information was provided in the original case report, authorization from the Ethics Committee was not required for this study.

\section{Results}

Using the search terminology, 38 case reports were identified from our database search, covering the period from January 2008 to February 2021. Three patients were excluded due to lack of age and CSF protein concentration, respectively. One case report was excluded because it was written in Japanese. Two case reports were not included because the diagnosis of GBS was ambiguous due to a disease progression similar to acute-onset CIDP. In addition, two patients with a history of GBS had no serious toxicities or deterioration of the previous autoimmune disorders after ICI therapy.
The two patients were also excluded. A total of 30 case reports (total patients $=33$ ) were used for final analysis [1342]. According to the diagnostic criteria of GBS in NINDS, all the above 33 cases were consistent with the features of GBS. The clinical data and diagnostic details of all included patients were summarized in Tables 1-3.

3.1. Demographic Characteristics. GBS cases $(n=33)$ were from the United State $(n=13)$, United Kingdom $(n=3)$, Japan $(n=2)$, Italy $(n=2)$, Belgium $(n=2)$, Australia $(n=2$ ) , China $(n=1)$, Greece $(n=1)$, Netherlands $(n=4)$, France $(n=2)$, and German $(n=1)$ (Table 1$)$. Of the 33 cases, twenty-six of these cases were male and seven were female, with an average of $62.2 \pm 11.1$ years (median: 65 years and range: 37-81 years). There was a male preponderance in 33 ICI-associated GBS patients we collected, with 3.7 times as many cases as female ( 26 vs. 7 cases: $78.8 \%$ vs. $21.2 \%$ ). There was a significant difference between male and female ages at onset (mean: $64.4 \pm 10.3$ vs. $54.1 \pm 11.1$ years, $P=0.0278$ ). The reports of comorbidities were variable, and no epidemics of specific diseases had been observed, so we did not analyze the comorbidities.

3.2. Immune Checkpoint Inhibitors and Tumor Type. Of ICIassociated GBS patients, sixteen patients exposed to nivolumab, eleven patients were investigated with ipilimumab, and seven patients were treated with pembrolizumab. Of these, four patients received nivolumab in combination with ipilimumab, and one patient received nivolumab in combination with pembrolizumab. In the above cases, the types of tumor the patients suffered from were melanoma $(n=20)$ $[13,14,16,17,23,24,28,29,31-37,39-42]$, lung tumor $(n=7)[18,21,25,26,28,30,38]$, urinary tumor $(n=5)$ $[13,15,19,20,22]$, and nasal cancer $(n=1)$ [27]. The details were summarized in Tables 4 and 5 .

3.3. Clinical Features of GBS Spectrum. The onset time of symptoms in 32 patients was analyzed, and the results suggested that the median time was 8.2 weeks after the initiation of ICI treatments. The minimum was 0.7 weeks (5 days) [37], and the maximum was 59 weeks [19]. As shown in Table 1, the time to neurological plateau was mentioned in 23 cases, with an average of $16.0 \pm 8.4$ days (range 7-44 days). The most common manifestations of GBS associated with ICIs were weakness $(93.9 \%, 31 / 33)$, hyporeflexia or areflexia $(90.9 \%$, $30 / 33)$, and paresthesia $(81.8 \%, 27 / 33)$. Among them, sensory symptoms and paraparesis or tetraparesis cooccurred in $45.5 \%$ (15/33) of cases. Other less common symptoms included cranial nerve involvement $(20.7 \%, 7 / 33)$, dysphagia or dysarthria $(18.2 \%, 6 / 33)$, respiratory symptoms $(21.2 \%, 6 / 33)$, and ataxia $(9.1 \%, 3 / 33)$. There were also 2 rare cases of GBS patients with dysautonomia as the onset symptoms $[17,36]$. The Hughes Functional Grading Scale (HFGS) [43] was used to evaluate the clinical severity, and higher numbers indicated more severe disability. The average was $3.85 \pm 1.42$, with a high score of 6 and a low score of 1 (Table 2).

3.4. Electrophysiological, CSF, and Imaging Results. Twentyseven patients underwent electrophysiological examination, and 18 cases had detailed electrophysiological reports. In 


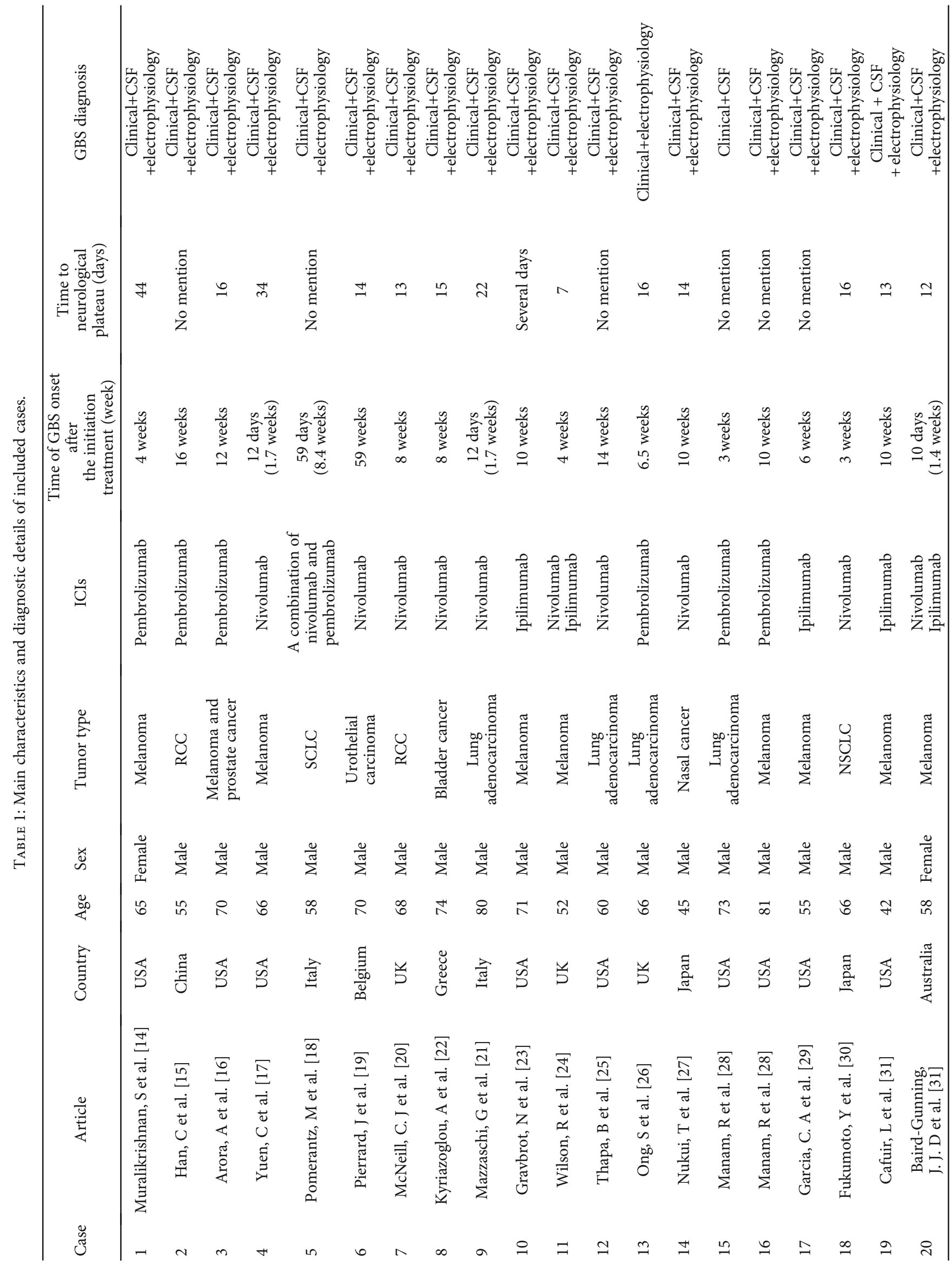




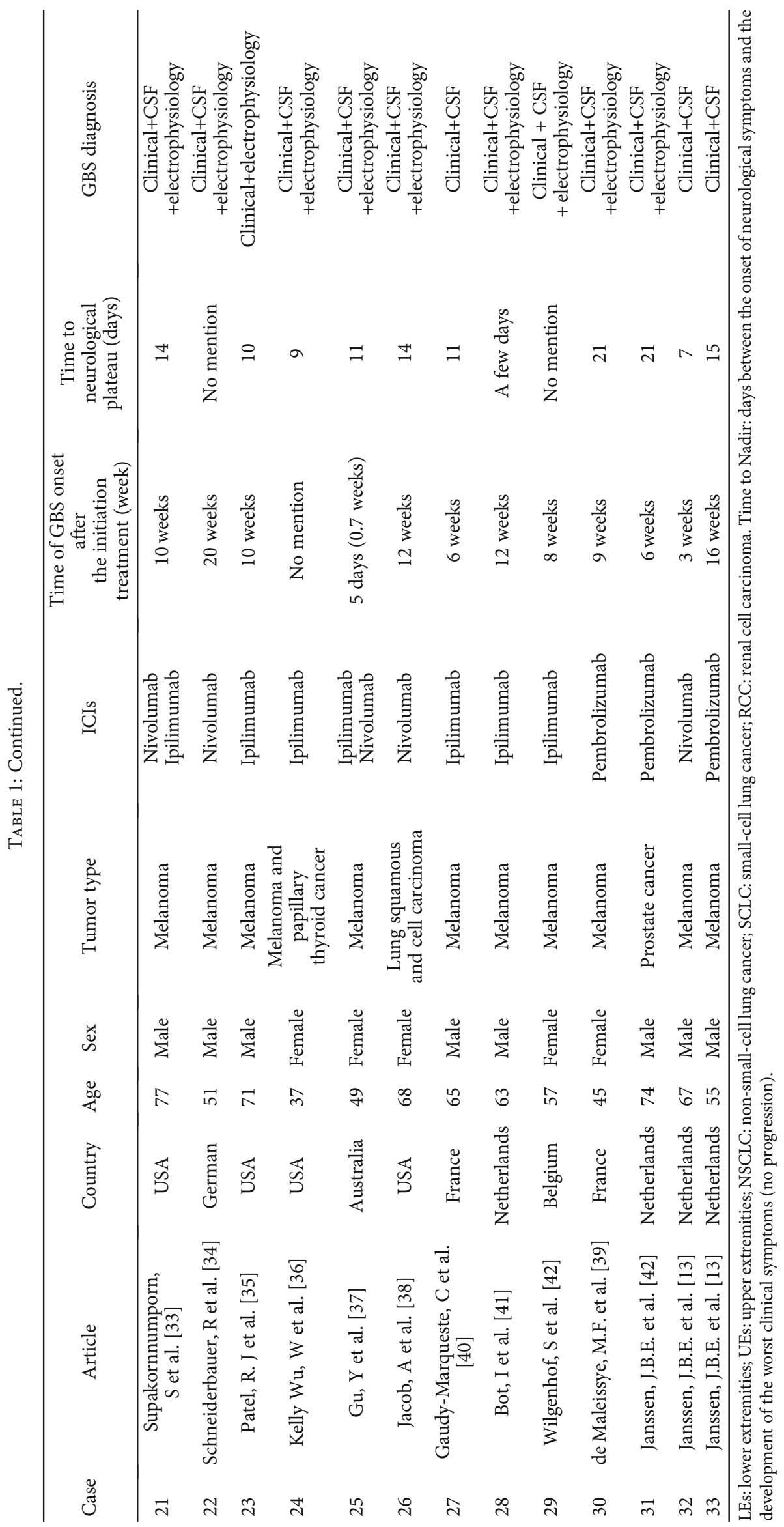




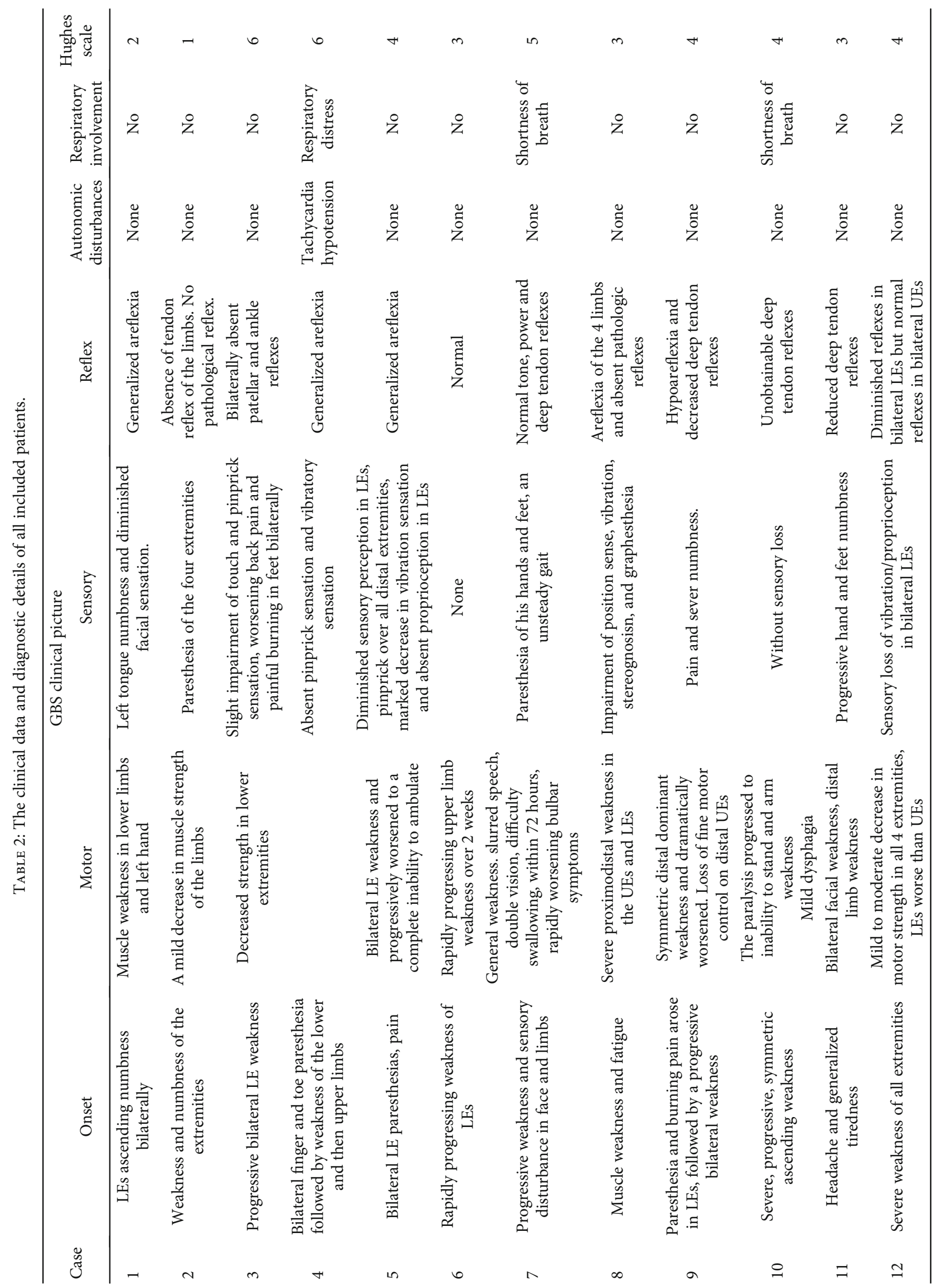




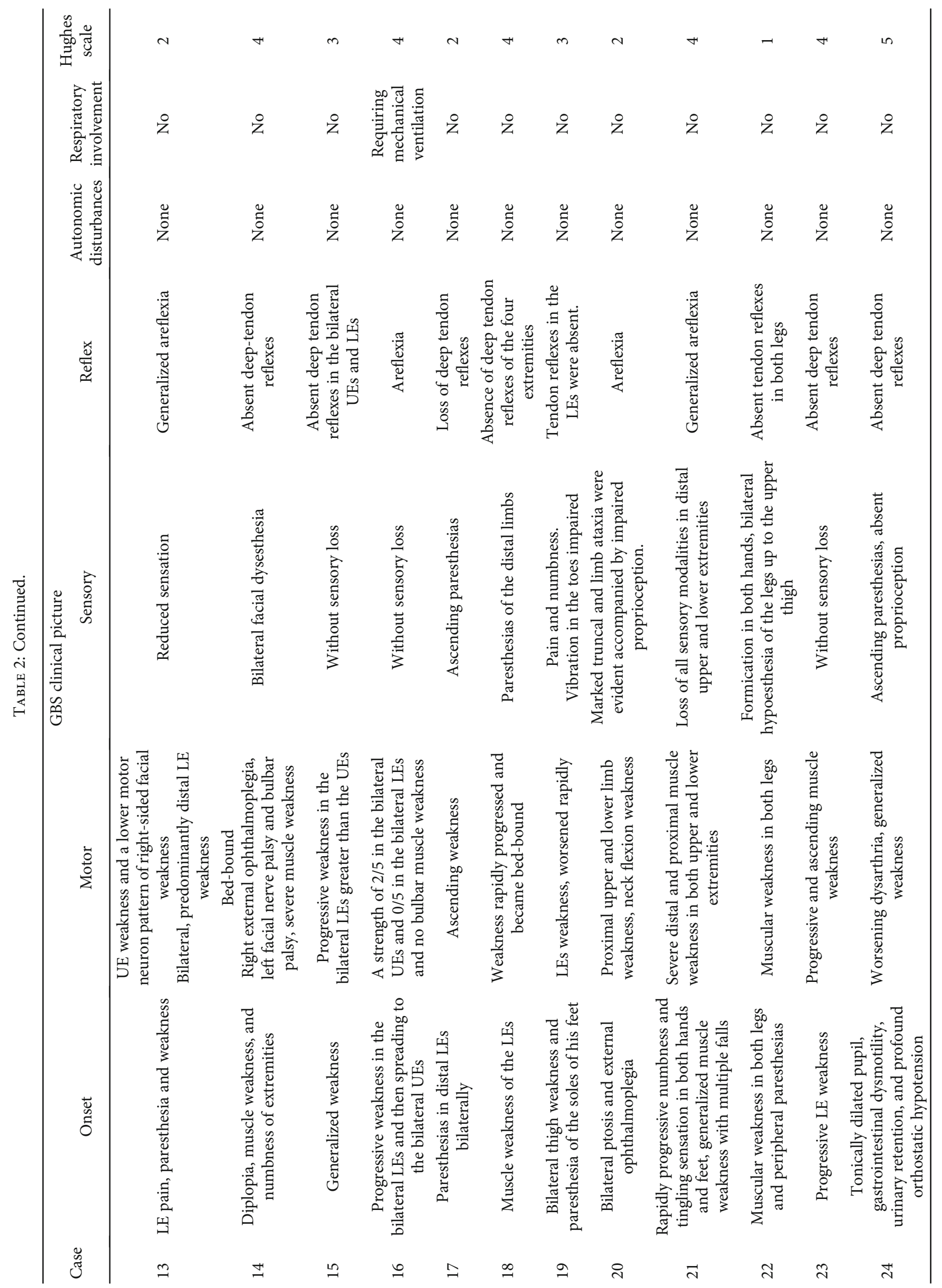




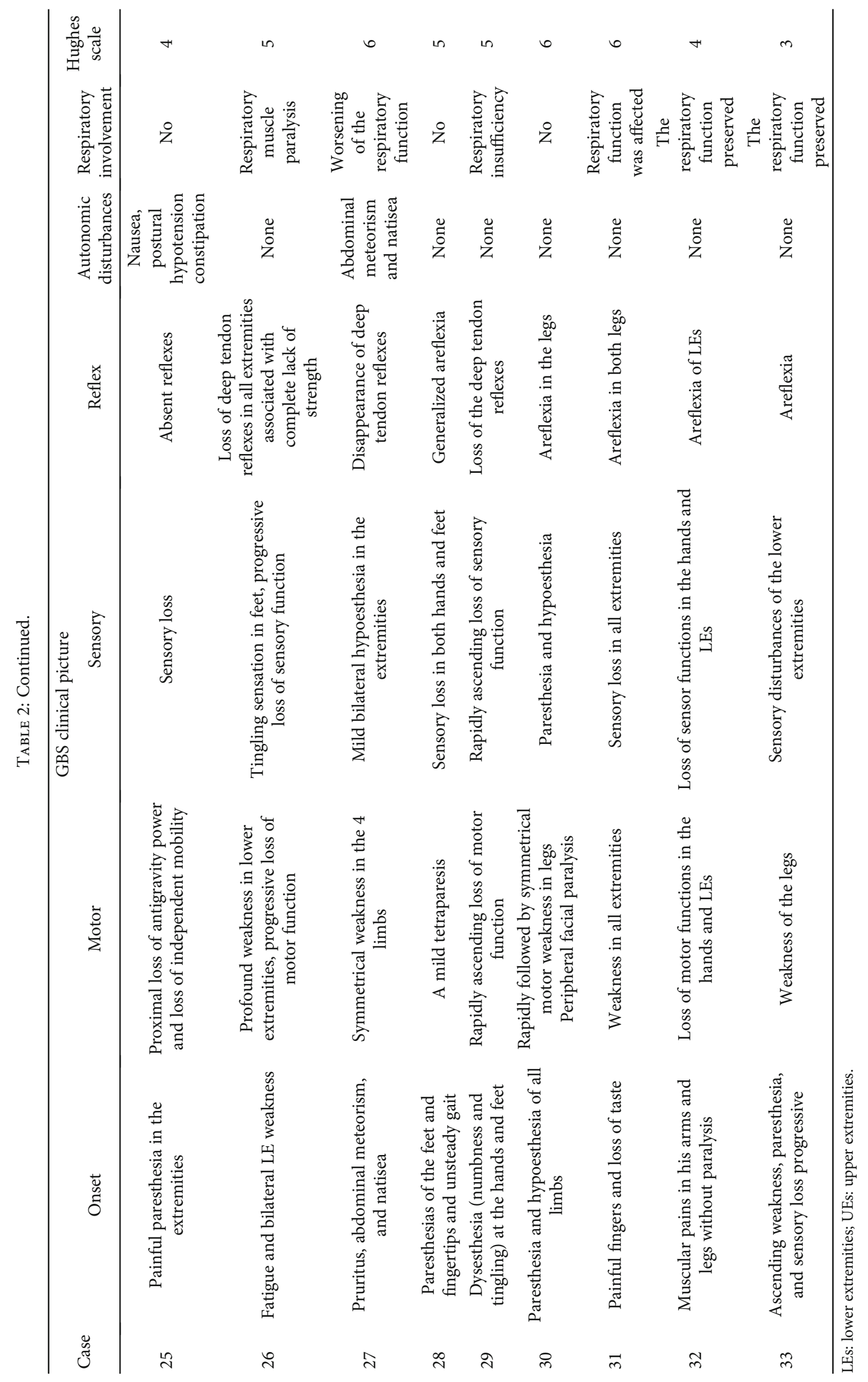




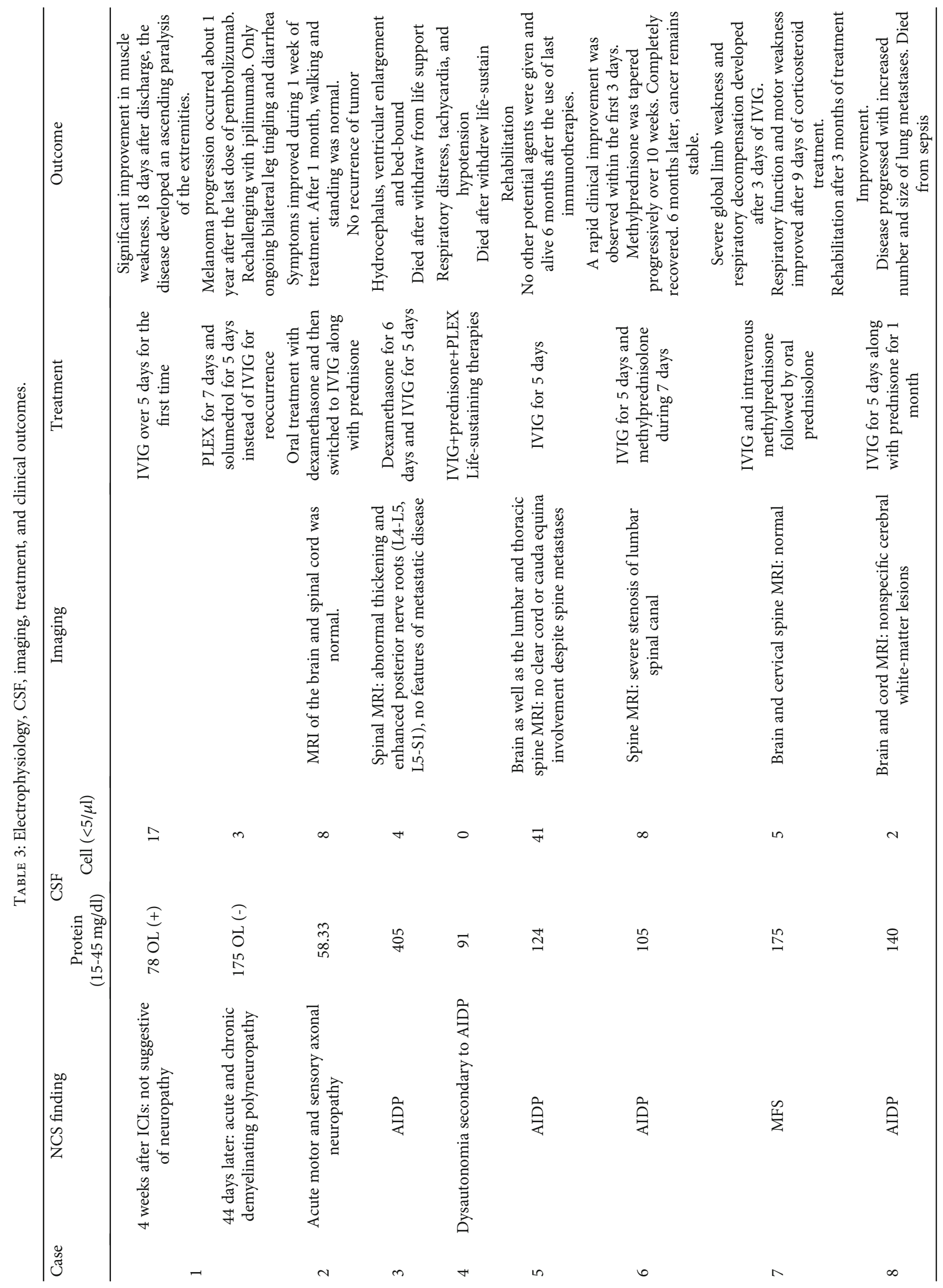




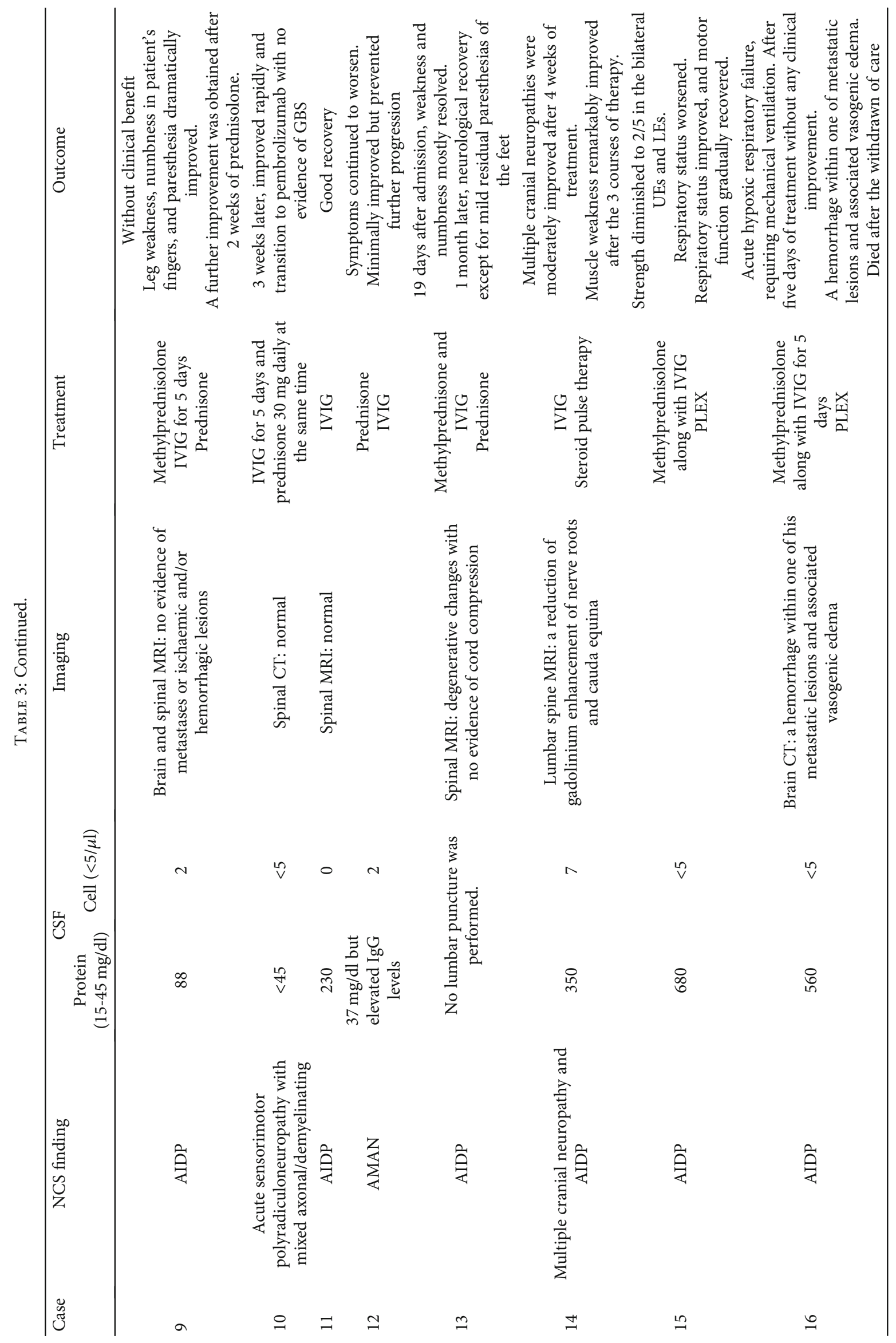




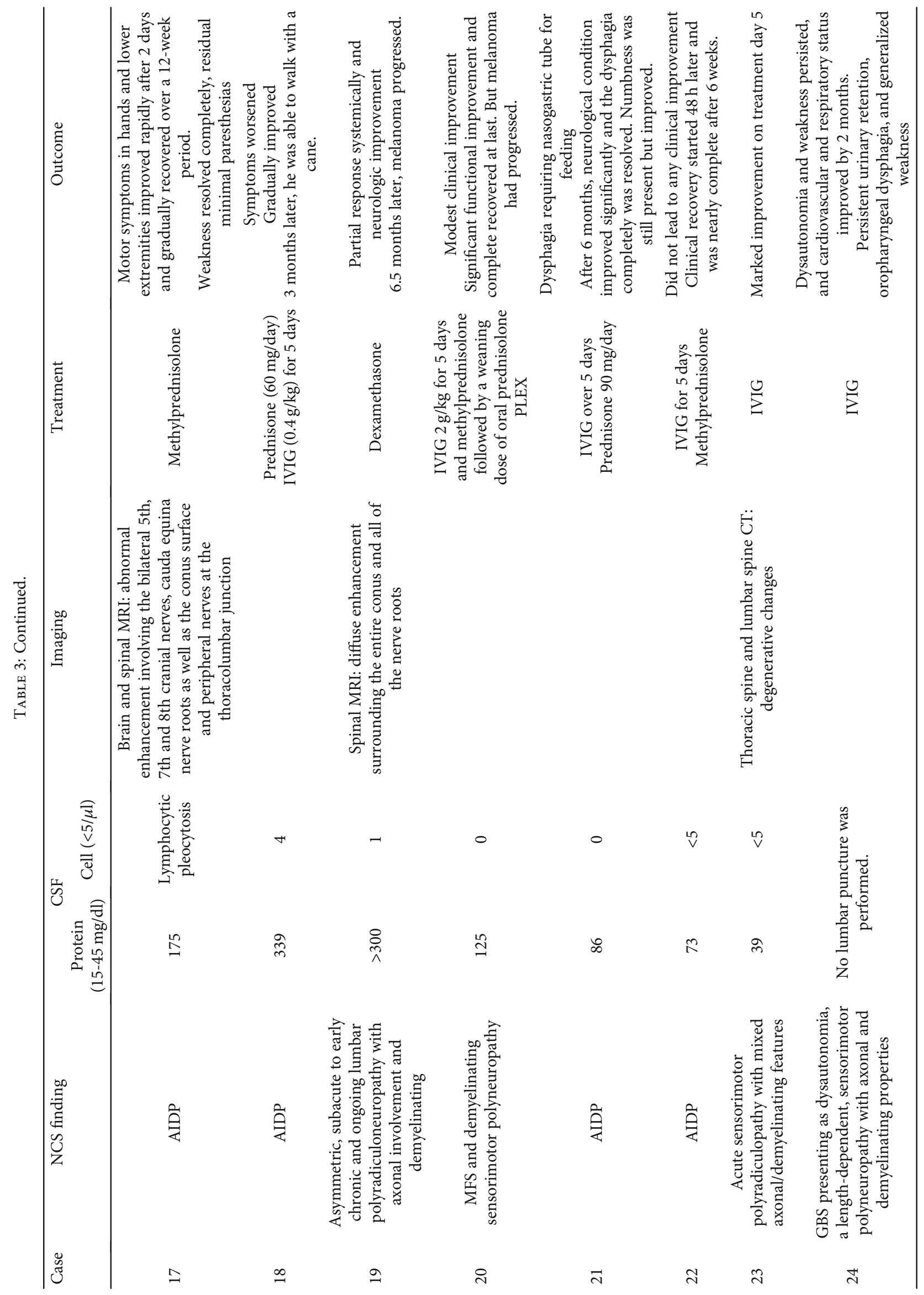




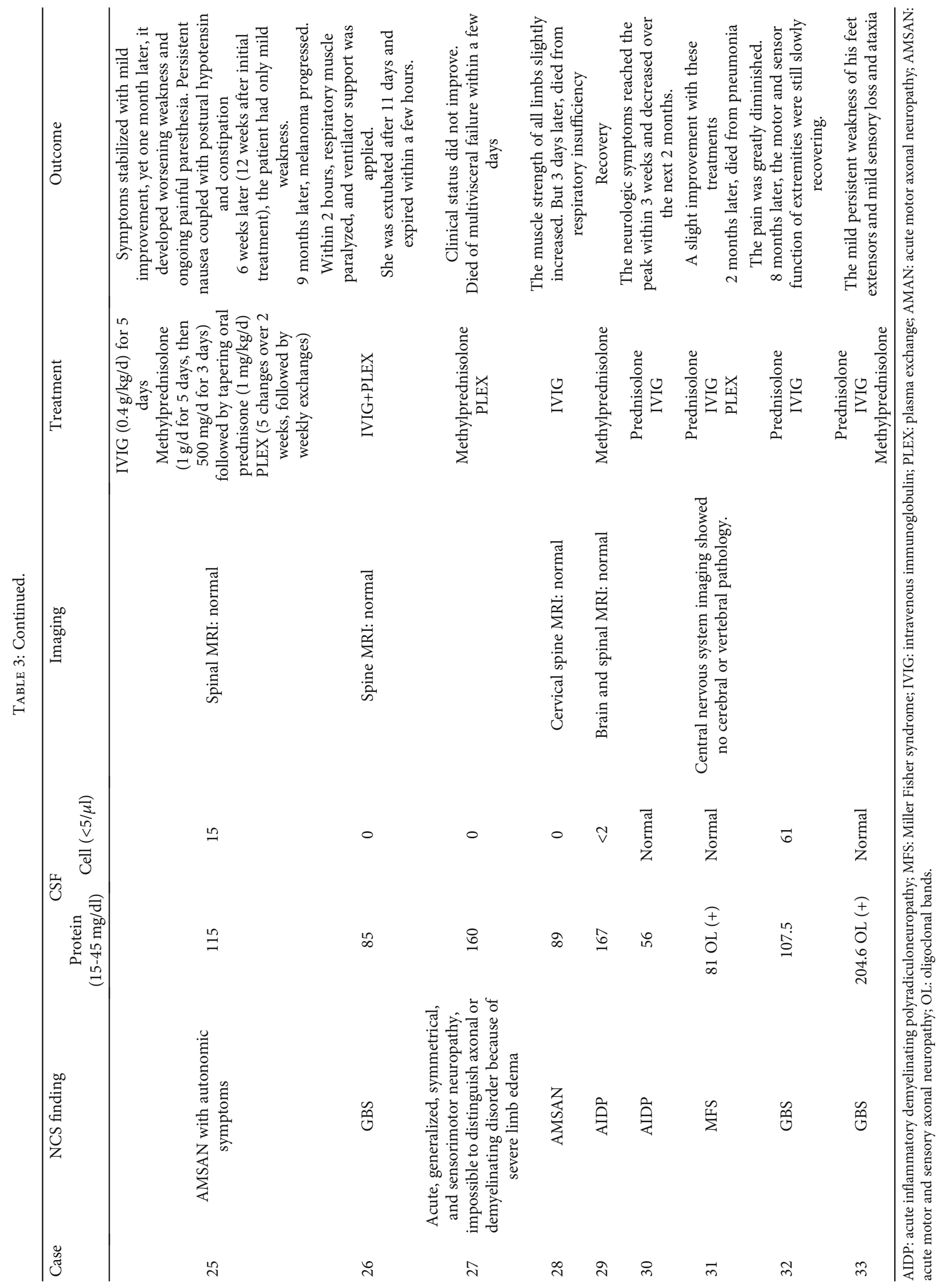




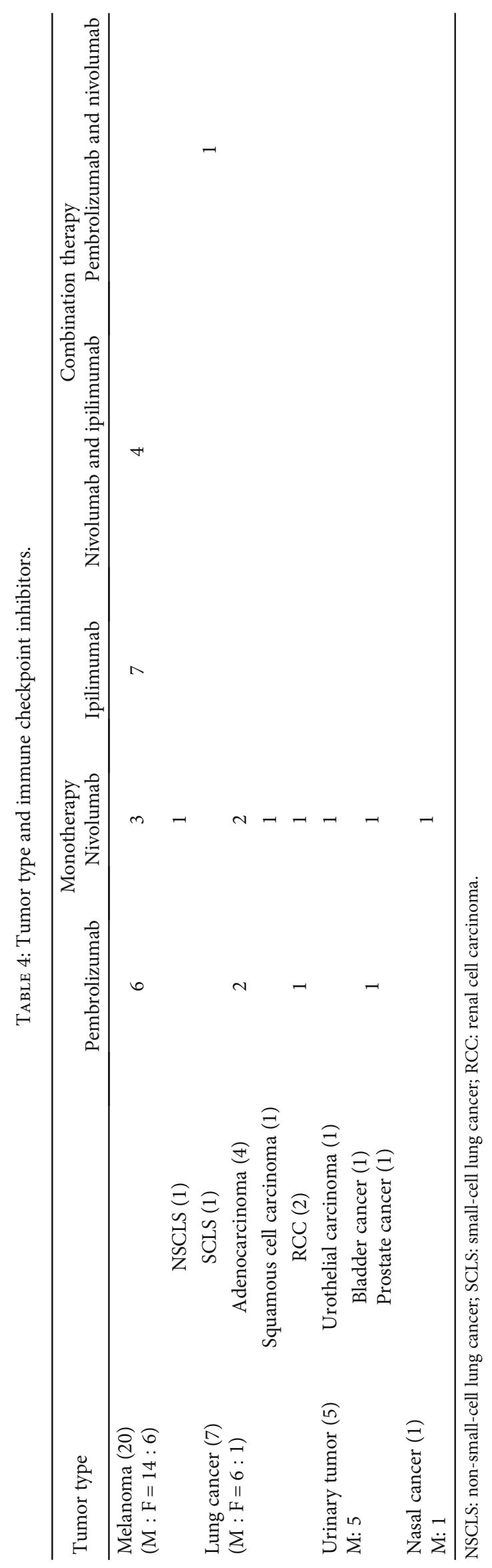


TABLE 5: Treatment and prognosis of ICIs-GBS.

\begin{tabular}{lccc}
\hline Treatment $(n=33)$ & Recovery & $\begin{array}{c}\text { Progression } \\
\text { Residual } \\
\text { dysfunction }\end{array}$ & Death \\
\hline IVIG $(n=3)$ & 2 & 6 & 1 \\
IVIG+steroid $(n=16)$ & 8 & & 2 \\
IVIG+PLEX $(n=1)$ & 2 & 2 & 1 \\
IVIG+steroid+PLEX & 1 & 3 & 3 \\
$(n=7)$ & 13 & 11 & 1 \\
$\begin{array}{l}\text { Steroid }(n=5) \\
\text { Steroid+PLEX }(n=1)\end{array}$ & & & 1 \\
Total & 13 & 9 \\
\hline
\end{tabular}

IVIG: intravenous immunoglobulin; PLEX: plasma exchange.

the remaining cases, only the GBS subtype was mentioned. $54.5 \%(18 / 33)$ of cases were consistent with AIDP [16-19, $21,22,24,26-30,33,34,39,42]$ and axonal damage accounted for $12.1 \%$ (4/33, AMSAN: 3 and AMAN: 1$)$ [15, $23,25,27]$. The subtypes in four cases were equivocal because they showed mixed features of axonal injury and demyelination $[23,31,35,36]$. There were also three cases, two with MFS [13, 20] and the other with MFS-GBS overlap [32]. Regrettably, there were two cases of GBS diagnosis with no mention of subtypes $[13,38,40]$.

CSF analysis was performed in 31 out of the 33 cases. The average protein level of CSF in patients with GBS related to ICIs was $180.68 \pm 152.51 \mathrm{mg} / \mathrm{dl}$ (range: $37-680 \mathrm{mg} / \mathrm{dl}$ ). $63.6 \%$ of the cases $(21 / 33)$ presented with typical albuminocytological dissociation (cells $\leq 5 / \mu \mathrm{l}$ and CSF protein $>45$ $\mathrm{mg} / \mathrm{dl})[14,16,20-22,24,28,30-34,38,40-42]$. The median value of CSF protein was $124.5 \mathrm{mg} / \mathrm{dl}$ (range: $73-680 \mathrm{mg} / \mathrm{dl}$ ). Slight pleocytosis (i.e., cell count $>5 / \mu \mathrm{l}$ ) was detected in $4 / 33$ cases $(12.1 \%)$ with a maximum cell count of $15 / \mu l$ and a median CSF protein of $115 \mathrm{mg} / \mathrm{dl}[15,19,27,37]$. CSF protein values and cell counts were normal in three cases [23, 25, 35 ], and there was elevated CSF immunoglobulin G levels in one case [25].

MRI was performed in $54.5 \%$ (18/33) of cases, of which both brain and spinal cord were examined in 8 cases and only spinal cord was 10 cases. MRI results showed nerve root involvement in 4 cases [16, 27, 29, 31], severe spinal stenosis in 1 case [19], cranial nerve involvement in 1 case, and normal or no metastatic signs in the rest [29].

3.5. Treatment and Prognosis of GBS. Twenty-seven cases $(81.8 \%)$ were treated with intravenous immunoglobulin (IVIG), sixteen of the 27 patients with a combination of steroid therapy and one with PLEX. Seven of the 27 patients were treated with all three treatments. Five cases were treated with steroid therapy alone, and one case was treated with steroid combined with PLEX. No patients were treated with PLEX alone.

After treatment, 24 cases (72.7\%) recovered or had only mild residual dysfunction, with two of them rechallenged ipilimumab [14] or pembrolizumab [21], and 5 cases had residual mild paresthesia $[13,14,26,29,33]$. Among the 33 ICISrelated GBS patients, 5 patients developed tumor progression after discontinuation of ICIS treatment, including 4 patients with melanoma [14, 31, 32, 37] and 1 patient with bladder cancer [22]. Of the eight deaths, three died of the respiratory muscle paralysis caused by GBS [17, 38, 41], 2 died of infection $[13,39]$, and three were due to progression of the tumor caused by ICI discontinuation $[16,28,40]$.

\section{Discussion}

The effects of checkpoint inhibition affect a wide range of system and trigger a wide range of autoimmune toxicity. The exact mechanism of neurological irAEs in ICI-treated patients is unknown [44]. The existence of shared antigens between the tumor and itself may be one possible mechanism, such as gangliosides found in both melanoma and Schwann cells [45]. ICI-related GBS is rare as a type of neurological irAEs, but it can develop into life-threatening consequence once occurred. Diagnosis of ICI-related GBS should be made in the shortest possible time so as not to delay the administration of immune-regulation therapy.

There was a male preponderance in 33 ICI-related GBS patients we collected, with 3.71 times as many cases as female (male 26 and female 7). However, among GBS triggered by infection, males were affected only 1.5 times more frequently than females [11]. An epidemiological study on the risk factors of melanoma showed that the incidence of melanoma of men was almost three times that of by women by the age of 75 [12]. The incidence of lung tumor in men has historically been higher than in women, although the incidence of lung tumor in women has risen since the 1960s, especially in younger women [46-48]. Perhaps the higher incidence of cancer in men, resulting in more opportunities for men to use ICIs, was one of the reasons for the higher incidence of ICI-related GBS. Besides, ICIs were more effective for male cancer patients than female patients [49]. And the development of irAEs was related to the beneficial effects of immunotherapy in malignant tumors, especially in advanced-stage melanoma, advanced and metastatic NSCLC, and advanced renal cell carcinoma (RCC) [50]. The high effectiveness of ICI treatment in male patients may be also responsible for the male preponderance in ICI-related GBS.

In this study, melanoma had the largest number of tumor type that caused GBS after ICI treatment. Neurological irAEs were rare, with an incidence of less than 3\% [8], whereas the overall incidence of severe nerve injury in melanoma patients treated with nivolumab with or without ipilimumab reached $0.93 \%$ [7], nearly one-third of the total neurological irAEs. Well know, GBS is a multifactorial autoimmune disorder, cell-mediated immunity plays an important role in immunopathology of all types of GBS. The activation of T cell caused by bacterial and virus leads to the production of cytokines and the release of free radicals, thus resulting in segmental demyelination [51]. And the cross-reaction between B-cell autoantibodies and axon gangliosides leads to axonal degradation [51]. Melanocytes and Schwann cells originate from the neural crest and have many common epitopes in humoral and cellular immune responses [52]. $\mathrm{T}$ cells regulate the degree of initial response of $\mathrm{T}$ cells by upregulating CTLA4 , while PD-1 inhibits the response of $\mathrm{T}$ cells in peripheral 
tissues and plays an important role in immune self-tolerance [53]. Due to the cross-reaction of molecular mimicry, T cellmediated autoimmunity against melanoma cell antigens may also have an effect on the myelin antigens on Schwann cell membranes. In addition, the response rate of melanoma to ICIs was higher than other tumors [54]. A FAERS database-based clinical study also showed that patients with melanoma or non-small-cell lung cancer maybe at higher risk of fatal neurologic AEs [55]. Therefore, the author speculated that melanoma patients were more likely to develop GBS after ICI treatment than other types of tumors.

Existing reports suggested that the overall incidence of neurological adverse events (nAEs) at all levels was 3.8\% for anti-CTLA-4 and 6.1\% for anti-PD-1/PD-L1 [56]. CTLA-4 and its ligands are only expressed on immune cells, while PD-1 and PD-L1 (ligands of PD-1) are expressed on both immune and nonimmune cells in peripheral tissues [57]. The difference in spatial distribution between the CTLA-4 and PD-1 pathways may explain the high incidence of GBS in patients treated with nivolumab.

Many literatures had reported the timing of irAEs onset after the initiation of ICI treatments. The skin manifestations appeared at 2-3 weeks, and immune-mediated colitis, hepatitis, pneumonitis, and nephritis appeared approximately 5-10 weeks, 12-16 weeks, 8-14 weeks, and 14-42 weeks, respectively. Endocrine dysfunctions appeared from 9 weeks [58]. At present, there is no literature on the time to onset of neurologic symptom related to GBS caused by ICIs. The initial time of symptoms in 33 patients in this article was analyzed, and the results suggested that the median time was 8.2 weeks after the initiation of ICI treatments. Compared with GBS caused by infectious triggers, neurologic symptom appeared much later. This conclusion was expected to be helpful for the rapid diagnosis of ICIs-GBS.

Of the 33 GBS related to ICI patients we collected, the initial symptoms were very similar to infection-triggered GBS. Lumbar puncture was performed, and CSF analysis revealed elevated protein levels and albuminocytologic dissociation. A study of 962 patients with infection-induced GBS showed that the average protein level was $113.8 \pm$ $11.8 \mathrm{mg} / \mathrm{dl}$ (range: $18-450 \mathrm{mg} / \mathrm{dl}$ ) [59]. In this study, the average protein level of CSF in patients with GBS related to ICIs was $180.68 \pm 152.51 \mathrm{mg} / \mathrm{dl}$ (range: $37-680 \mathrm{mg} / \mathrm{dl}$ ). From a numerical point of view, the levels of CSF protein level of ICI-related GBS seemed to be higher than those of GBS induced by infection. Although it was not possible to distinguish infection-induced GBS from ICI-induced GBS based on CSF protein levels, lumbar puncture and protein-cell-separation were valuable in differentiating a variety of diseases such as spinal cord compression, metabolic diseases, side effects of drugs, vasculitis, and chronic inflammatory demyelinating polyneuropathy. In addition to elevated protein levels, lymphocytosis may also occur [37]. A similar pattern was observed in this study, with 4 patients showing a mild lymphocytosis in the CSF. However, multiple cases had been reported of lymphocytosis in the CSF of patients with infection-induced GBS [60-63]. Obviously, lymphocytosis in the CSF was not a characteristic of ICI-related GBS.
The electrophysiological results detailed in this study indicated that ICI-related GBS was a generalized, sensorimotor polyneuropathy characterized by mixed axonal/demyelination. An electrophysiological study of ICI-related peripheral neuropathy showed that immune-mediated neuropathy mainly manifested as demyelination of motor nerves, followed by length dependent axonal loss in sensory nerve [60]. The results of this study were in part consistent with the above conclusions. No matter what kind of electrophysiological changes were closely related to the autoimmune response of ICIs against peripheral nerve tissue, the onset of symptoms of neuropathy was closely related to ICI treatment, and corticosteroid or immune-regulatory therapy had good results.

In the treatment of all 33 patients, the immediate discontinuation of ICIs was an uncontroversial decision. Most patients received IVIG after discontinuation, with 16 patients receiving both IVIG and steroid therapy. Despite steroids were generally not recommended for treatment in infection-induced GBS, in ICI-related GBS, both American Society of Clinical Oncology (ASCO) Clinical Practice Guideline and National Comprehensive Cancer Network (NCCN) guidelines stated that trial of (methyl) prednisolone $1-2 \mathrm{mg} / \mathrm{Kg}$ was reasonable $[64,65]$, especially when CSF pleocytosis was higher than being anticipated for GBS [10]. And if the symptoms deteriorated, plasma exchange or IVIG treatment could be considered [66].

Among the 33 ICI-related GBS patients, 5 patients developed tumor progression after discontinuation of ICI treatment. One of the four patients with progressed melanoma rechallenged different class of immunotherapy, with significant and sustained response nearly 1 year later and no recurrence of GBS-like neuropathy. Whether or not to retreat with ICIs and whether to retreat with the same or different ICIs are challenges for oncologists. There is also limited data on the clinical efficacy and safety of retreatment. The current guidelines suggested that corticoid therapy and temporary or permanent discontinuing ICIs were required for grade $\geq$ 2 irAEs and permanently discontinuing ICI treatment for grade 4 irAEs $[65,67]$. A study had shown that the recurrence rate of the same irAEs resulting in discontinuation of ICI treatment in cancer patients who rechallenged the same ICI was $28.6 \%$ (anti-PD-1 or anti-PD-L1 monotherapy), $47.4 \%$ (anti-CTLA-4 monotherapy), and 43.5\% (combination therapy), respectively [66]. The recurrence rate of irAEs varied depending on the organ involved in the initial irAEs, with gastrointestinal irAEs having the highest recurrence rate [68]. And the variables associated with a higher recurrence rate of irAEs were anti-CTLA-4 regimen, age, colitis, hepatitis, and pneumonia, in order [68]. In addition, the duration from ICI discontinuation to rechallenge, and the severity of the initial irAEs did not predict whether irAEs would reappear after rechallenge of ICIs [69]. However, no relevant literature has been reported on whether discontinuation due to ICI-related GBS can rechallenge the same or different ICIs again.

In addition, paraneoplastic peripheral neuropathy should be excluded in the diagnosis of ICI-related GBS. Paraneoplastic peripheral neuropathy is a remote effect of the malignancy 
mediated by the immune system. It develops prior or during a cancer and is independent of tumor infiltration or cancer therapy [70]. Paraneoplastic sensory neuropathy is the most frequent in this group of disorders, and motor, autonomic, or central nervous systems are also involved [71]. Date on the treatment of paraneoplastic peripheral neuropathy is limited, and the combination of malignancy therapy with immunomodulatory therapies such as corticosteroids, IV immunoglobulin, or immunosuppressants may be effective [72]. However, ICI-related GBS occurred in malignant tumors after ICI treatment, and the immunomodulatory therapeutic effect was obvious. This is the most obvious difference between the two.

Here are also several limitations in this study. First, the number of the included cases was small which limited us to perform subgroup analysis. Second, patients with various cancers were included, which might have bias in the incidence of some adverse effects.

\section{Conclusion}

Elderly male patients with melanoma were most likely to develop ICI-related GBS. And the median duration was 8.2 weeks after the initial ICI treatment. In order to make a final diagnosis, physicians need to collect specific neurological symptoms and signs and combine them with CSF analysis and electrophysiological examination. In addition, imaging is required to exclude tumor metastasis. Immediate termination of ICIs followed by IVIG in combination with high-dose steroids therapy or PLEX and supportive treatment could lead to a better prognosis.

\section{Abbreviations}

ICIs: Immune checkpoint inhibitors

CTLA-4: Cytotoxic T lymphocyte-associated antigen-4

PD-1: $\quad$ Programmed cell death protein-1

PD-L1: Programmed cell death protein-1 ligand

irAEs: Immune-related adverse events

GBS: $\quad$ Guillain-Barré syndrome

AIDP: Acute inflammatory demyelinating

polyneuropathy

AMAN: Acute motor axonal neuropathy

AMSAN: Acute motor and sensory axonal neuropathy

MFS: $\quad$ Miller fisher syndrome

DML: Distal motor latency

EMG: Needle electromyography

IVIG: Intravenous immunoglobulin

PLEX: Plasma exchange

CSF: $\quad$ Cerebrospinal fluid

CNS: Central nervous system

NSCLC: Non-small-cell lung cancer

RCC: Renal cell carcinoma.

\section{Conflicts of Interest}

The authors have no conflicts of interest to declare.

\section{References}

[1] D. Psimaras, R. Velasco, C. Birzu et al., "Immune checkpoint inhibitors-induced neuromuscular toxicity: from pathogenesis to treatment," Journal of the peripheral nervous system : JPNS, vol. 24, no. 2, pp. S74-S85, 2019.

[2] J. D. Wolchok, H. Kluger, M. K. Callahan et al., "Nivolumab plus ipilimumab in advanced melanoma," The New England Journal of Medicine, vol. 369, no. 2, pp. 122-133, 2013.

[3] P. Schmid, S. Adams, H. S. Rugo et al., "Atezolizumab and nab-paclitaxel in advanced triple-negative breast cancer," The New England Journal of Medicine, vol. 379, no. 22, pp. 21082121, 2018.

[4] A. Markham and S. Duggan, "Cemiplimab: first global approval,” Drugs, vol. 78, no. 17, pp. 1841-1846, 2018.

[5] K. M. Hargadon, C. E. Johnson, and C. J. Williams, "Immune checkpoint blockade therapy for cancer: an overview of FDAapproved immune checkpoint inhibitors," International Immunopharmacology, vol. 62, pp. 29-39, 2018.

[6] L. A. Emens, P. A. Ascierto, P. K. Darcy et al., "Cancer immunotherapy: opportunities and challenges in the rapidly evolving clinical landscape," European journal of cancer, vol. 81, pp. 116-129, 2017.

[7] L. B. Kennedy and A. K. S. Salama, "A review of cancer immunotherapy toxicity," CA: a Cancer Journal for Clinicians, vol. 70, no. 2, pp. 86-104, 2020.

[8] M. Touat, D. Talmasov, D. Ricard, and D. Psimaras, "Neurological toxicities associated with immune-checkpoint inhibitors," Current Opinion in Neurology, vol. 30, no. 6, pp. 659668, 2017.

[9] B. R. Wakerley and N. Yuki, "Infectious and noninfectious triggers in Guillain-Barré syndrome," Expert Review of Clinical Immunology, vol. 9, no. 7, pp. 627-639, 2013.

[10] C. Astaras, R. de Micheli, B. Moura, T. Hundsberger, and A. F. Hottinger, "Neurological adverse events associated with immune checkpoint inhibitors: diagnosis and management," Current Neurology and Neuroscience Reports, vol. 18, no. 1, p. 3, 2018.

[11] H. Yoshikawa, "Epidemiology of Guillain-Barré Syndrome," Brain and nerve, vol. 67, no. 11, pp. 1305-1311, 2015.

[12] M. Rastrelli, S. Tropea, C. R. Rossi, and M. Alaibac, "Melanoma: epidemiology, risk factors, pathogenesis, diagnosis and classification," In vivo (Athens, Greece), vol. 28, no. 6, pp. 1005-1011, 2014.

[13] J. B. E. Janssen, T. Y. S. Leow, K. H. Herbschleb et al., "Immune checkpoint inhibitor-related Guillain-Barré syndrome: a case series and review of the literature," Journal of immunotherapy (Hagerstown, Md: 1997), 2021.

[14] S. Muralikrishnan, L. K. Ronan, S. Coker, P. K. Rauschkolb, and K. Shirai, "Treatment considerations for patients with unresectable metastatic melanoma who develop pembrolizumab-induced Guillain-Barré toxicity: a case report," Case reports in oncology, vol. 13, no. 1, pp. 43-48, 2020.

[15] C. Han, J. A. Ma, Y. Zhang, Y. Jiang, C. Hu, and Y. Wu, "Guillain-Barre syndrome induced by pembrolizumab and sunitinib: a case report," Molecular and Clinical Oncology, vol. 13, no. 1, pp. 38-42, 2020.

[16] A. Arora, A. Shere, K. Patel, N. Gunawardhana, and E. LiFuentes, "A rare case of autoimmune demyelinating polyneuropathy and hydrocephalus secondary to pembrolizumab," 
Journal of Investigative Medicine High Impact Case Reports, vol. 8, p. 2324709620916358, 2020.

[17] C. Yuen, D. Kamson, B. Soliven, C. Kramer, F. Goldenberg, and K. Rezania, "Severe relapse of vaccine-induced GuillainBarré syndrome after treatment with nivolumab," Journal of Clinical Neuromuscular Disease, vol. 20, no. 4, pp. 194-199, 2019.

[18] M. Pomerantz, D. A. Lichtenstein, I. Niesvizky-Kogan, R. Sohal, J. Leibovitch, and A. Basnet, "Nivolumab and ipilimumab-induced acute inflammatory demyelinating polyradiculoneuropathy: a case report," Journal of immunotherapy (Hagerstown, Md : 1997), vol. 42, no. 9, pp. 348-349, 2019.

[19] J. Pierrard, B. Petit, S. Lejeune, and E. Seront, "Isolated adrenocorticotropic hormone (ACTH) deficiency and Guillain-Barré syndrome occurring in a patient treated with nivolumab," BML Case Reports, vol. 12, no. 8, 2019.

[20] C. J. McNeill, J. Fehmi, and J. Gladwin, "Price C: A rare case of Miller Fisher variant of Guillain-Barré syndrome (GBS) induced by a checkpoint inhibitor," BML Case Reports, vol. 12, no. 8, 2019.

[21] G. Mazzaschi, P. Bordi, R. Fioretzaki et al., "Nivolumabinduced Guillain-Barre syndrome coupled with remarkable disease response in a case of heavily pretreated lung adenocarcinoma," Clinical Lung Cancer, 2019.

[22] A. Kyriazoglou, M. Liontos, C. Papadopoulos et al., "Guillain-Barre syndrome related to nivolumab: case report of a patient with urothelial cancer and review of the literature," Clinical Genitourinary Cancer, vol. 17, no. 2, pp. e360e364, 2019.

[23] N. Gravbrot, K. Scherer, and S. Sundararajan, "Safe Transition to Pembrolizumab following Ipilimumab-Induced GuillainBarré Syndrome: A Case Report and Review of the Literature," Case Reports in Oncological Medicine, vol. 2019, 5 pages, 2019.

[24] R. Wilson, D. A. Menassa, A. J. Davies et al., "Seronegative antibody-mediated neurology after immune checkpoint inhibitors," Annals of Clinical Translational Neurology, vol. 5, no. 5, pp. 640-645, 2018.

[25] B. Thapa, S. Khalid, R. Vakili, J. Ui, and S. Misbah, "Nivolumab-associated Guillain-Barre syndrome in a patient with non-small-cell lung cancer," American Journal of Therapeutics, vol. 25, no. 6, pp. e761-e763, 2018.

[26] S. Ong, J. Chapman, G. Young, and T. Mansy, "Guillain-Barrelike syndrome during pembrolizumab treatment," Muscle \& Nerve, 2018.

[27] T. Nukui, Y. Nakayama, M. Yamamoto et al., "Nivolumabinduced acute demyelinating polyradiculoneuropathy mimicking Guillain-Barré syndrome," Journal of the Neurological Sciences, vol. 390, pp. 115-116, 2018.

[28] R. Manam, J. L. Martin, J. A. Gross et al., "Case reports of pembrolizumab-induced acute inflammatory demyelinating polyneuropathy," Cureus, vol. 10, no. 9, article e3371, 2018.

[29] C. A. Garcia, A. El-Ali, T. J. Rath et al., "Neurologic immunerelated adverse events associated with adjuvant ipilimumab: report of two cases," Journal for Immunotherapy of Cancer, vol. 6, no. 1, p. 83, 2018.

[30] Y. Fukumoto, M. Kuwahara, S. Kawai, K. Nakahama, and S. Kusunoki, "Acute demyelinating polyneuropathy induced by nivolumab," Journal of Neurology, Neurosurgery, and Psychiatry, vol. 89, no. 4, pp. 435-437, 2018.

[31] L. Cafuir, D. Lawson, N. Desai, V. Kesner, and A. Voloschin, "Inflammatory demyelinating polyneuropathy versus lepto- meningeal disease following Ipilimumab," Journal for Immunotherapy of Cancer, vol. 6, no. 1, p. 11, 2018.

[32] J. J. D. Baird-Gunning, D. Weerasinghe, M. Silsby et al., "Miller fisher syndrome associated with immunotherapy for metastatic melanoma," The Neurohospitalist, vol. 8, no. 4, pp. 191-193, 2018.

[33] S. Supakornnumporn and B. Katirji, "Guillain-Barré syndrome triggered by immune checkpoint inhibitors: a case report and literature review," Journal of Clinical Neuromuscular Disease, vol. 19, no. 2, pp. 80-83, 2017.

[34] R. Schneiderbauer, M. Schneiderbauer, W. Wick, A. H. Enk, H. A. Haenssle, and J. C. Hassel, "PD-1 antibody-induced Guillain-Barré syndrome in a patient with metastatic melanoma," Acta Dermato-Venereologica, vol. 97, no. 3, pp. 395396, 2017.

[35] R. J. Patel, M. A. Liu, A. Amaraneni, and S. K. Sindhu, "Rare side effect of adjuvant ipilimumab after surgical resection of melanoma: Guillain-Barré syndrome," BMJ Case Reports, vol. 2017, 2017.

[36] W. Kelly Wu, K. K. Broman, E. R. Brownie, and R. M. Kauffmann, "Ipilimumab-induced Guillain-Barré syndrome presenting as dysautonomia: an unusual presentation of a rare complication of immunotherapy," Journal of immunotherapy, vol. 40, no. 5, pp. 196-199, 2017.

[37] Y. Gu, A. M. Menzies, G. V. Long, S. L. Fernando, and G. Herkes, "Immune mediated neuropathy following checkpoint immunotherapy," Journal of clinical neuroscience : official journal of the Neurosurgical Society of Australasia, vol. 45, pp. 14-17, 2017.

[38] A. Jacob, D. C. Unnikrishnan, A. Mathew, B. Thyagarajan, and S. Patel, "A case of fatal Guillain-Barre syndrome from antiPD1 monoclonal antibody use," Journal of Cancer Research and Clinical Oncology, vol. 142, no. 8, pp. 1869-1870, 2016.

[39] M. F. de Maleissye, G. Nicolas, and P. Saiag, "Pembrolizumabinduced demyelinating polyradiculoneuropathy," The New England Journal of Medicine, vol. 375, no. 3, pp. 296-297, 2016.

[40] C. Gaudy-Marqueste, S. Monestier, J. Franques, E. Cantais, M. A. Richard, and J. J. Grob, "A severe case of ipilimumabinduced guillain-barré syndrome revealed by an occlusive enteric neuropathy: a differential diagnosis for ipilimumabinduced colitis," Journal of immunotherapy, vol. 36, no. 1, pp. 77-78, 2013.

[41] I. Bot, C. U. Blank, W. Boogerd, and D. Brandsma, "Neurological immune-related adverse events of ipilimumab," Practical Neurology, vol. 13, no. 4, pp. 278-280, 2013.

[42] S. Wilgenhof and B. Neyns, "Anti-CTLA-4 antibody-induced Guillain-Barré syndrome in a melanoma patient," Annals of oncology : official journal of the European Society for Medical Oncology, vol. 22, no. 4, pp. 991-993, 2011.

[43] M. Shi, J. Zhu, and H. Deng, "Clinical characteristics of intravenous injection of monosialotetrahexosyl ganglioside sodium-related Guillain-Barre syndrome," Frontiers in Neurology, vol. 10, p. 225, 2019.

[44] M. Eltobgy, H. Oweira, U. Petrausch et al., "Immune-related neurological toxicities among solid tumor patients treated with immune checkpoint inhibitors: a systematic review," Expert Review of Neurotherapeutics, vol. 17, no. 7, pp. 725-736, 2017.

[45] P. C. Pan and A. Haggiagi, "Neurologic immune-related adverse events associated with immune checkpoint inhibition," Current Oncology Reports, vol. 21, no. 12, p. 108, 2019. 
[46] D. Twardella, K. Geiss, M. Radespiel-Tröger, A. Benner, J. H. Ficker, and M. Meyer, "Trends in incidence of lung cancer according to histological subtype among men and women in Germany : analysis of cancer registry data with the application of multiple imputation techniques," Federal Health Gazette, Health Research, Health Protection, vol. 61, no. 1, pp. 20-31, 2018.

[47] L. A. Torre, R. L. Siegel, and A. Jemal, "Lung cancer statistics," Advances in Experimental Medicine and Biology, vol. 893, pp. 1-19, 2016.

[48] R. Meza, C. Meernik, J. Jeon, and M. L. Cote, "Lung cancer incidence trends by gender, race and histology in the United States, 1973-2010," PLoS One, vol. 10, no. 3, article e0121323, 2015.

[49] S. Wang, L. A. Cowley, and X. S. Liu, "Sex differences in cancer immunotherapy efficacy, biomarkers, and therapeutic strategy," Molecules, vol. 24, no. 18, 2019.

[50] O. Fiala, O. Sorejs, J. Sustr, R. Kucera, O. Topolcan, and J. Finek, "Immune-related adverse effects and outcome of patients with cancer treated with immune checkpoint inhibitors," Anticancer Research, vol. 40, no. 3, pp. 1219-1227, 2020.

[51] Z. Ebrahim Soltani, F. Rahmani, and N. Rezaei, "Autoimmunity and cytokines in Guillain-Barré syndrome revisited: review of pathomechanisms with an eye on therapeutic options," European Cytokine Network, vol. 30, no. 1, pp. 1$14,2019$.

[52] W. Metcalfe, J. Anderson, V. A. Trinh, and W. J. Hwu, “Antiprogrammed cell death-1 (PD-1) monoclonal antibodies in treating advanced melanoma," Discovery Medicine, vol. 19, no. 106, pp. 393-401, 2015.

[53] L. Chen and D. B. Flies, "Molecular mechanisms of T cell costimulation and co-inhibition," Nature Reviews Immunology, vol. 13, no. 4, pp. 227-242, 2013.

[54] M. Sade-Feldman, K. Yizhak, S. L. Bjorgaard et al., "Defining T cell states associated with response to checkpoint immunotherapy in melanoma," Cell, vol. 176, no. 1-2, p. 404, 2019.

[55] T. Mikami, B. Liaw, M. Asada et al., "Neuroimmunological adverse events associated with immune checkpoint inhibitor: a retrospective, pharmacovigilance study using FAERS database," Journal of Neuro-Oncology, vol. 152, no. 1, pp. 135144, 2021.

[56] C. Perrinjaquet, N. Desbaillets, and A. F. Hottinger, "Neurotoxicity associated with cancer immunotherapy: immune checkpoint inhibitors and chimeric antigen receptor T-cell therapy," Current Opinion in Neurology, vol. 32, no. 3, pp. 500-510, 2019.

[57] A. Cortellini, S. Buti, V. Agostinelli, and M. Bersanelli, "A systematic review on the emerging association between the occurrence of immune-related adverse events and clinical outcomes with checkpoint inhibitors in advanced cancer patients," Seminars in Oncology, vol. 46, no. 4-5, pp. 362-371, 2019.

[58] V. Kumar, N. Chaudhary, M. Garg, C. S. Floudas, P. Soni, and A. B. Chandra, "Current diagnosis and management of immune related adverse events (irAEs) induced by immune checkpoint inhibitor therapy," Frontiers in Pharmacology, vol. 8, p. 49, 2017.

[59] S. R. Sudulagunta, M. B. Sodalagunta, M. Sepehrar et al., "Guillain-Barré syndrome: clinical profile and management," German medical science, vol. 13, 2015.

[60] G. T. Doctor, S. K. Alexander, and A. Radunovic, "GuillainBarré syndrome with exaggerated pleocytosis and anti-GM1 ganglioside antibodies," BML Case Reports, vol. 2018, 2018.
[61] M. Dy, R. L. Leshner, and J. R. Crawford, "An unusual case of recurrent Guillain-Barre syndrome of a different subtype five years after initial diagnosis," Case reports in neurological medicine, vol. 2013, 4 pages, 2013.

[62] H. Rauschka, K. Jellinger, H. Lassmann, F. Braier, and M. Schmidbauer, "Guillain-Barré syndrome with marked pleocytosis or a significant proportion of polymorphonuclear granulocytes in the cerebrospinal fluid: neuropathological investigation of five cases and review of differential diagnoses," European Journal of Neurology, vol. 10, no. 5, pp. 479-486, 2003.

[63] J. Berciano, J. Figols, A. García et al., "Fulminant GuillainBarré syndrome with universal inexcitability of peripheral nerves: a clinicopathological study," Muscle \& Nerve, vol. 20, no. 7, pp. 846-857, 1997.

[64] J. A. Thompson, B. J. Schneider, J. Brahmer et al., "Management of immunotherapy-related toxicities, version 1.2019," Journal of the National Comprehensive Cancer Network, vol. 17, no. 3, pp. 255-289, 2019.

[65] J. R. Brahmer, C. Lacchetti, B. J. Schneider et al., "Management of immune-related adverse events in patients treated with immune checkpoint inhibitor therapy: American Society of Clinical Oncology clinical practice guideline," Journal of Clinical Oncology : Official Journal of the American Society of Clinical Oncology, vol. 36, no. 17, pp. 1714-1768, 2018.

[66] J. Haanen, F. Carbonnel, C. Robert et al., "Management of toxicities from immunotherapy: ESMO Clinical Practice Guidelines for diagnosis, treatment and follow-up," Annals of oncology : official journal of the European Society for Medical Oncology, vol. 28, no. 4, pp. iv119-iv142, 2017.

[67] J. Shi, J. Niu, D. Shen et al., "Clinical diagnosis and treatment recommendations for immune checkpoint inhibitor-related adverse reactions in the nervous system," Thorac Cancer, vol. 11, no. 2, pp. 481-487, 2020.

[68] C. Dolladille, S. Ederhy, M. Sassier et al., "Immune checkpoint inhibitor rechallenge after immune-related adverse events in patients with cancer," JAMA Oncology, vol. 6, no. 6, pp. 865871,2020

[69] M. Allouchery, T. Lombard, M. Martin et al., "Puyade M: Safety of immune checkpoint inhibitor rechallenge after discontinuation for grade $\geq 2$ immune-related adverse events in patients with cancer," Journal for Immunotherapy of Cancer, vol. 8 , no. $2,2020$.

[70] J. C. Antoine and J. P. Camdessanché, "Paraneoplastic neuropathies," Current Opinion in Neurology, vol. 30, no. 5, pp. 513520, 2017.

[71] S. A. Rudnicki and J. Dalmau, "Paraneoplastic syndromes of the peripheral nerves," Current Opinion in Neurology, vol. 18, no. 5, pp. 598-603, 2005.

[72] S. Muppidi and S. Vernino, "Paraneoplastic neuropathies," CONTINUUM: Lifelong Learning in Neurology, vol. 20, no. 5, pp. 1359-1372, 2014. 